\title{
Perturbação de Jogos de Internet: Revisão da Evidência Científica
}

\section{Internet Gaming Disorder: State of the Art}

Diogo Beirão¹, Tiago Sousa², Paula Assunção ${ }^{3}$, Abílio Malheiro4 ${ }^{4}$ Diana Gonzaga ${ }^{5}$

Autor Correspondente/Corresponding Author:

Diogo Beirão [diogobeirao10@gmail.com]

Rua São Salvador, 9, 4100-464 Porto, Portugal

ORCID iD: 0000-0001-5612-8941

\section{RESUMO}

A Internet permitiu a difusão dos videojogos, facilitando o seu uso desregulado. A perturbação de jogos de Internet traduz o uso com impacto nas áreas funcionais e sociais. A utilização crescente de tecnologias conduz a um aumento da prevalência deste distúrbio.

O nosso objetivo foi efetuar uma atualização da evidência científica sobre perturbação de jogos de Internet.

Foi efetuada uma pesquisa de artigos científicos publicados entre janeiro de 2013 e fevereiro de 2019, em inglês, português e espanhol. As palavras-chave utilizadas foram "behavior, addictive", "video games" e "Internet".

Podemos concluir que a perturbação de jogos de Internet é mais prevalente no sexo masculino e no continente asiático e subcontinente norte-americano. Os principais critérios de diagnóstico têm permitido consolidá-la como uma perturbação distinta. Os estudos neuroimagiológicos têm comprovado alterações nas áreas de controlo executivo e sistema da recompensa. As terapêuticas farmacológicas e não farmacológicas revelam-se promissoras, apesar de não ser clara a eficácia direta ou sobre as comorbilidades associadas.

PALAVRAS-CHAVE: Comportamento Aditivo; Internet; Jogos de Vídeo

1. Interno de Formação Específica de Medicina Geral e Familiar da USF Ramalde, Porto, Portugal. 2. Interno de Formação Específica de Medicina Geral e Familiar da USF S. João do Porto, Porto, Portugal. 3. Assistente de Medicina Geral e Familiar da USF Ramalde, Porto, Portugal. 4. Assistente graduado de Medicina Geral e Familiar da USF S. João do Porto, Porto, Portugal. 5. Assistente Hospitalar de Pediatria - Unidade de Neurodesenvolvimento do Centro Materno Infantil do Norte, Centro Hospitalar Universitário do Porto, Porto, Portugal. 


\section{ABSTRACT}

The Internet contributed for the widespread of videogames, allowing its unregulated use. Internet gaming disorder defines the negative impact of said use on functional areas of life. The increasing use of technology heralds higher prevalence of this disorder.

Our objective was to review the state of the art on Internet gaming disorder.

We searched for scientific papers published between January of 2013 and February of 2019 in English, Portuguese and Spanish. The MeSH terms used were "behavior, addictive", "video games" and "Internet".

We can conclude that Internet gaming disorder is most prevalent amongst males, and in the Asian continent and North American subcontinent. The main diagnostic criteria have strengthened Internet gaming disorder as a distinct disorder. Neuroimagiologic studies confirm the deficit on executive control areas and reward system. Pharmacological and non-pharmacological therapies look promising, though it is not clear if it targets Internet gaming disorder primarily or its comorbidities.

KEYWORDS: Behavior, Addictive; Internet; Video Games

\section{INTRODUÇÃO}

Os videojogos são um meio de entretenimento com várias décadas de existência. ${ }^{1}$ Contudo, o desenvolvimento da Internet e da World Wide Web em 1989 permitiu que se integrassem no nosso quotidiano pela fácil e rápida disponibilidade de serviços e modificação dos padrões de interação social. ${ }^{2}$ A indústria dos videojogos acompanhou esta mudança através da transição de um formato de jogador único para um de jogadores múltiplos em mundos virtuais partilhados. No entanto, apesar de constituir um novo meio de comunicação interpessoal, as suas características fomentam o seu uso desmedido, que permite uma fuga da realidade. Atualmente, mais de 90\% das crianças e adolescentes dos Estados Unidos da América jogam videojogos, sendo que parte destas irá desenvolver sintomas que se poderão enquadrar na perturbação de jogos de Internet (PJI). 3,4

Este artigo tem como objetivo abordar a evidência científica desta perturbação através da exploração do seu diagnóstico, epidemiologia, neurofisiologia e programas terapêuticos propostos.

\section{MÉTODOS}

Foram pesquisadas as bases de dados National Guideline Clearinghouse, National Electronic Library for Health, Canadian Medical Association Practice Guidelines Infobase, The Cochrane Library, Database of Abstracts of Reviews of Effects, Bandolier e Evidence based medicine online, a 12 de fevereiro de 2019, com uso dos termos MeSH "video games", "behavior, addictive" e/ou "Internet". Foram consultados os critérios de diagnóstico do Manual de Diagnóstico e Estatística das Perturbações Mentais, atualmente na sua $5^{a}$ edição (DSM-5), e da Classificação Internacional de Doenças, presentemente na sua $11^{2}$ revisão (CID-11).
Os critérios de inclusão estabelecidos foram a tipologia de artigo definida como norma de orientação clínica, meta-análise, revisão sistemática ou estudo original; artigos publicados em inglês, português ou espanhol; data de publicação entre 1 de janeiro de 2013 até à data da pesquisa.

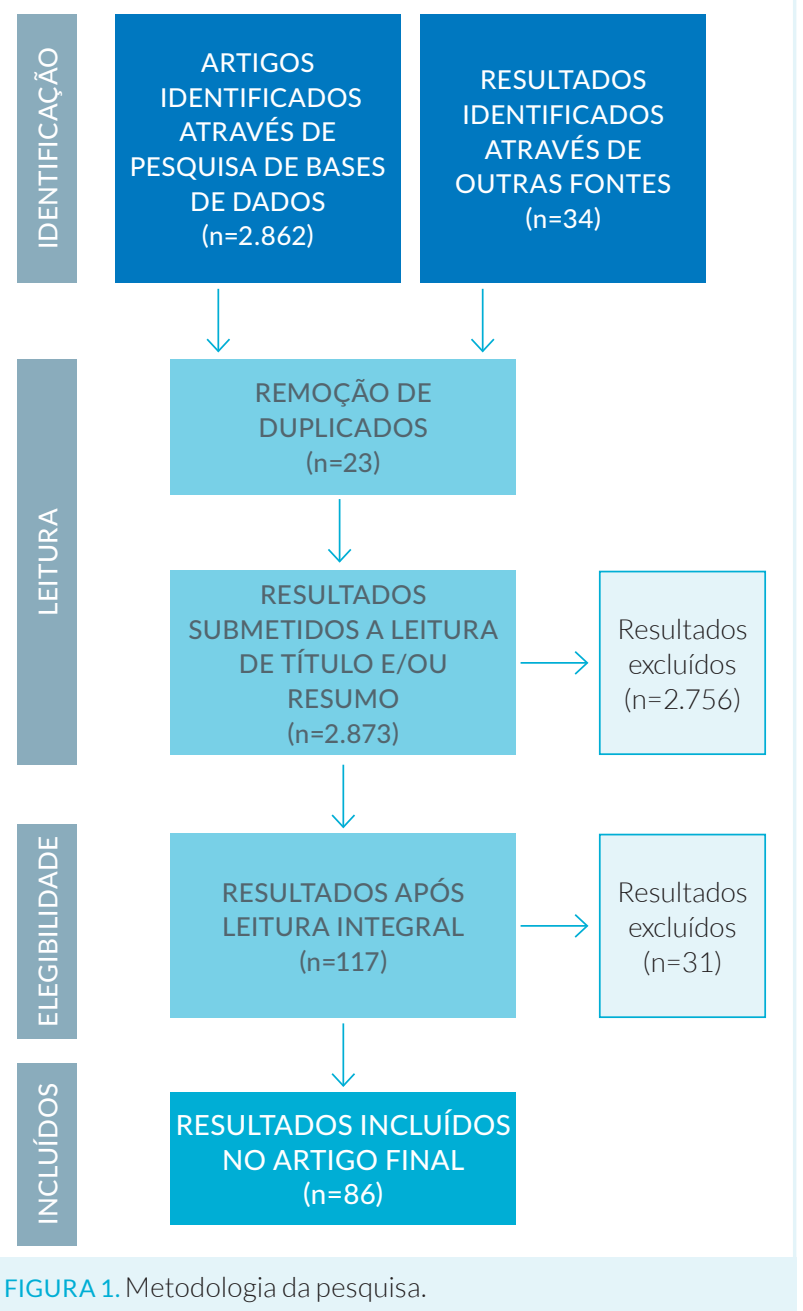


Foram excluídos artigos considerados não pertinentes pelos autores após a leitura do título, do resumo ou leitura integral.

Dos 2896 resultados obtidos, 23 resultados foram excluídos por duplicação, 2756 resultados foram excluídos após leitura do título e/ou resumo, 31 resultados foram excluídos após leitura integral, tendo sido incluídos 52 resultados. A metodologia da pesquisa encontra-se explanada na Fig. 1. Adicionalmente, foram incluídas 34 referências secundárias, citadas na literatura original ou pesquisadas para esclarecimento de tópicos específicos.

\section{DIAGNÓSTICO}

Em 2013, a DSM-5 definiu a PJI como "uso persistente e recorrente da Internet para envolvimento em jogos, com frequência com outros jogadores, que leva a défices ou mal-estar clinicamente significativos". ${ }^{5}$ É diagnosticada a partir do momento em que se verifica a presença de pelo menos 5 dos 9 critérios, durante pelo menos 12 meses (Tabela 1). Ao tomar esta iniciativa, a American Psychiatric Association (APA) identificou a PJI como uma entidade independente de outros distúrbios relacionados com jogos e/ou Internet. Desta forma, esta definição exclui dependências relacionadas com jogo e conteúdos sexuais e o uso profissional da Internet. Contudo, esta entidade foi incluída na "Secção III - Medidas e Modelos Emergentes", na qual estão incluídas perturbações não oficializadas, cuja investigação é encorajada. ${ }^{5}$

Desde que a APA propôs estes critérios, surgiu o interesse em operacionalizá-los num instrumento de avaliação validado e universal. ${ }^{6-8}$ Até essa data, foram usadas várias ferramentas inespecíficas e direcionadas para o vício da Internet.9,10 Do conhecimento dos autores, até à data, foram desenvolvidos 9 inquéritos específicos para a PJI (Tabela 2), que diferem nas suas características e objetivos. ${ }^{11-19}$ Destes, apenas um dos inquéritos está validado para Portugal, o Internet Gaming Disorder Scale, short form, que está dirigido para a prática clínica. ${ }^{20}$

Mais recentemente, desde 2018, a CID-11 da Organização Mundial de Saúde contempla os distúrbios relacionados com jogos de computador, online e offline (Tabela 1). ${ }^{21}$ Tal como a definição do DSM-5, obriga a contextualizar a alteração do comportamento com o grau de impacto nas áreas pessoal, familiar, educacional, ocupacional, entre outras, de caráter contínuo ou recorrente. A definição da CID-11 também integra os 12 meses de duração do quadro para diagnóstico, mas prevê que esta duração possa ser encurtada, de acordo com a gravidade clínica.

TABELA 1. Comparação entre critérios de diagnóstico do DSM-5 e da CID-11.

\section{DSM-5}

Uso persistente e recorrente da Internet para envolvimento em jogos.

Défices ou mal-estar clinicamente significativos.

Presença de $\geq 5$ dos seguintes critérios num período de 12 meses:

1. Preocupação com jogos de Internet. ( $O$ indivíduo pensa sobre atividades de jogo prévias ou antecipa a realização do próximo jogo; jogar na Internet torna-se a atividade dominante da vida diária.)

2. Sintomas de abstinência quando os jogos de Internet são retirados. (Estes sintomas são tipicamente descritos como irritabilidade, ansiedade ou tristeza, mas não há sinais físicos de abstinência farmacológica.)

3. Tolerância - a necessidade de despender quantidades crescentes de tempo envolvido em jogos de Internet.

4. Tentativas mal sucedidas de controlar a participação em jogos de Internet.

5. Perda de interesse em passatempos e atividades de entretenimento prévias resultante de, e com a exceção do, uso de jogos de Internet.

6. Uso excessivo continuado de jogos de Internet apesar do conhecimento dos problemas psicossociais.

7. Ter enganado os membros da família, terapeutas ou outros relativamente à quantidade de jogo na Internet.

8. Uso de jogos de Internet para evitar ou aliviar um humor negativo (por exemplo, sentimentos de desesperança, culpa, ansiedade).

9. Ter arriscado ou perdido uma relação significativa, emprego ou oportunidades educacionais ou de carreira devido à participação em jogos de Internet.

\section{CID-11}

Padrão de comportamento contínuo ou episódico recorrente.

Padrão de comportamento com gravidade suficiente que resulta em compromisso pessoal, familiar, social, educacional, entre outras áreas importantes do funcionamento.

Características do comportamento evidentes durante pelo menos 12 meses (ou menos, se todos os critérios presentes e sintomatologia grave).

1. Compromisso do controlo com os videojogos (ex. início, frequência, intensidade, duração, finalização, contexto).

2. Prioridade crescente dos videojogos que atinge precedência relativamente a outros interesses e atividades diárias.

3. Prorrogação/Escalonamento dos videojogos apesar da ocorrência de consequências negativas. 
TABELA 2. Instrumentos de avaliação diagnóstica.

\begin{tabular}{|c|c|c|}
\hline Ferramenta & Descrição & $\begin{array}{l}\text { Validação } \\
\text { portuguesa }\end{array}$ \\
\hline IGD-20 (2014) & $\begin{array}{l}\text { Inquérito de auto-resposta referente às atividades de jogos de Internet nos últimos } 12 \text { meses, constituído } \\
\text { por } 20 \text { questões, cada uma com } 5 \text { opções graduadas de "discordo completamente" a "concordo completamente" } \\
\text { (pontuadas de } 1 \text { a 5), com um limiar diagnóstico de } 71 \text { pontos. }\end{array}$ & Não \\
\hline IGDS-SF9 $(2015)^{12}$ & $\begin{array}{l}\text { Inquérito de auto-resposta, referente às atividades de jogos de Internet nos últimos } 12 \text { meses, constituído por } \\
9 \text { questões, cada uma com } 5 \text { opções graduadas com "nunca" a "quase sempre" (pontuadas de } 1 \text { a } 5 \text { ), com um limiar } \\
\text { diagnóstico de } 36 \text { pontos. }\end{array}$ & $\operatorname{Sim}(2016)^{20}$ \\
\hline GAIT $(2015)^{13}$ & $\begin{array}{l}\text { Inquérito de auto-resposta, referente às atividades de jogos de Internet nos últimos } 12 \text { meses, constituído por } \\
15 \text { questões, cada uma com } 5 \text { opções graduadas com "discordo completamente" a "concordo completamente" } \\
\text { (pontuadas de } 0 \text { a 4). }\end{array}$ & Não \\
\hline $\begin{array}{l}\text { AICA-S-gaming } \\
(2015)^{14}\end{array}$ & $\begin{array}{l}\text { Inquérito de auto-resposta, referente às atividades de jogos de Internet nos últimos } 12 \text { meses, constituído por } \\
13 \text { questões, cada uma com } 5 \text { opções ou dicotómica, com classificação de "não problemático" (0 a } 6.5 \text { pontos ou pelo } \\
\text { menos } 1 \text { critério presente), "em risco" (7 a } 13 \text { pontos ou } 2 \text { a } 4 \text { critérios presentes) ou "uso aditivo indicando PJI" } \\
\text { (mais de } 13 \text { pontos ou } 5 \text { ou mais critérios presentes). }\end{array}$ & Não \\
\hline PIE-9 (2016) ${ }^{15}$ & $\begin{array}{l}\text { Inquérito de auto-resposta, referente às atividades de jogos de Internet nos últimos } 12 \text { meses, constituído por } \\
9 \text { questões, cada uma com } 5 \text { opções graduadas com "nunca" a "quase sempre" (pontuadas de } 1 \text { a 5), com um limiar } \\
\text { diagnóstico de pelo menos } 5 \text { questões pontuadas com } 4 \text { ou } 5 \text {. }\end{array}$ & Não \\
\hline IGD-Q (2016) 16 & $\begin{array}{l}\text { Inquérito de auto-resposta, referente às atividades de jogos de Internet nos últimos } 12 \text { meses, constituído por } \\
9 \text { questões dicotómicas (pontuadas em } 0 \text { ou 1), com um limiar diagnóstico de } 5 \text { pontos. }\end{array}$ & Não \\
\hline IGDT-10 (2017) & $\begin{array}{l}\text { Inquérito de auto-resposta, referente às atividades de jogos de Internet nos últimos } 12 \text { meses, constituído por } \\
10 \text { questões (as duas últimas valem por 1), cada uma com } 3 \text { opções com classificação de "nunca", "às vezes" } \\
\text { ou "frequentemente" (apenas este último valorizado com } 1 \text { ponto), com um limiar diagnóstico de } 5 \text { pontos. }\end{array}$ & Não \\
\hline SCI-IGD $(2017)^{18}$ & $\begin{array}{l}\text { Inquérito aplicado em entrevista, referente às atividades de jogos de Internet nos últimos } 6 \text { meses, constituído por } \\
12 \text { questões. }\end{array}$ & Não \\
\hline C-IGDS $(2017)^{19}$ & $\begin{array}{l}\text { Inquérito de auto-resposta, referente às atividades de jogos de Internet nos últimos } 12 \text { meses, constituído por } \\
9 \text { questões dicotómicas (pontuadas em } 0 \text { ou 1), com um limiar diagnóstico de } 5 \text { pontos. }\end{array}$ & Não \\
\hline
\end{tabular}

Legenda: $P J \mid=$ perturbação de jogos de Internet

\section{EPIDEMIOLOGIA}

Múltiplos estudos foram publicados na tentativa de objetivar a dimensão desta problemática. A prevalência global nos adolescentes é cerca de 4,6\%, sendo este valor variável entre estudos desde 0,6\% até 19,9\%. A comparação com os resultados obtidos ao nível das crianças $(4,2 \%)$ e dos adultos $(8,9 \%)$ revela um potencial início precoce da história natural desta patologia., 6,7

Relativamente ao sexo, verificou-se uma prevalência superior no sexo masculino (6,8\%) comparativamente ao sexo feminino (1,3\%), o que é compatível com o tipo de comportamento manifestado nesta patologia.,, 7

Ao longo das últimas 3 décadas, verificou-se uma redução progressiva da prevalência global de PJI. Contudo, este fenómeno deve ser interpretado de forma cuidadosa, uma vez que foram utilizados diferentes grupos de critérios nos vários estudos, diferente número de estudos ao longo das 3 décadas e intervalos de confiança alargados nos estudos da década de 90. Por este motivo, estes fatores poderão ter sobrestimado de forma errada a prevalência desta patologia na década de $90 .{ }^{6}$

A nível geográfico, verificaram-se prevalências mais elevadas ao nível do continente asiático e subcontinente norte-americano comparativamente ao continente europeu e Austrália. Este fenómeno pode ser explicado pela origem das principais produtoras de videojogos e pela proveniência da maior receita de videojogos. ${ }^{6,8}$

\section{NEUROFISIOLOGIA E CORRELAÇÃO COMPORTAMENTAL}

A investigação na área da PJI é limitada pela dependência em critérios de diagnóstico subjetivos, consequência da heterogeneidade que condiciona. ${ }^{22}$ Neste sentido, a investigação das alterações neuroimagiológicas tem permitido criar uma perspetiva mais objetiva desta perturbação e personalizar a terapêutica. A maioria dos estudos foca em investigação baseada em resultados de ressonância magnética nas suas formas estática e funcional, entre outras modalidades.

A PJI partilha não só características clínicas com outras perturbações aditivas, tais como desejo, abstinência e défices de controlo cognitivo, como também alterações semelhantes ao nível dos mecanismos neuronais, tais como circuitos dopaminérgicos e frontoestriados. ${ }^{23-27}$

Os indivíduos afetados pela PJI demonstram maior impulsividade, secundária a défices morfológicos e de atividade em estruturas cerebrais responsáveis pelo controlo inibitório e executivo. ${ }^{28-30}$ Apesar do córtex pré-frontal (CPF) ter o papel mais relevante, certas regiões do lobo parietal, ínsula, amígdala, córtex cingulado anterior, área motora suplementar, certas regiões do lobo temporal e ainda o cúneo também estão implicados, tal como a interligação entre estas áreas. ${ }^{31-40}$

Similar aos distúrbios de uso e dependência de substâncias, outra característica da PJ é a implicação no sistema 
de recompensa. ${ }^{29,41}$ Por oposição às alterações supracitadas, ocorre sobreativação de regiões cerebrais ligadas a este sistema perante estímulos relacionados com o jogo, tais como o estriado, o córtex orbitofrontal e córtices cingulados anterior e posterior. ${ }^{42}$ Também se verificaram anomalias nos circuitos frontoestriado e frontocingulado, que reforça a interligação entre antecipação da recompensa e maior impulsividade. ${ }^{43,44} \bigcirc$ aumento da densidade de matéria cinzenta no estriado ventral corrobora a maior sensibilidade destes indivíduos para a recompensa. ${ }^{44}$ Durante o jogo, ocorre libertação de dopamina comparável à utilização de outras substâncias, como a cocaína. ${ }^{29}$ A cronicidade deste processo está associada a alterações significativas da química cerebral das vias dopaminérgicas, com menor conectividade funcional e estrutural entre estruturas do sistema dopaminérgico em indivíduos com PJI. ${ }^{45,46}$

Além das alterações nos dois sistemas anteriormente referidos, outras funções encontram-se comprometidas na PJI. A adolescência é uma fase importante do desenvolvimento, onde discrepâncias entre a autoimagem e a imagem idealizada (pelo próprio ou outros) causam alterações com maior probabilidade de se repercutirem nas estruturas cerebrais. ${ }^{47-53}$ Os giros temporais médio e inferior à direita e esquerda, e o lóbulo parietal superior direito, implicados no reconhecimento facial e na orientação espacial, têm menor atividade em indivíduos afetados. ${ }^{54}$ A desregulação emocional pode ser explicada pela diminuição da densidade de matéria cinzenta nas amígdalas associada a um aumento da sua conectividade ao CPF e ínsula. ${ }^{55}$ Estudos em adultos jogadores de Massively Multiplayer Online Role-Playing Games (MMORPG) identificam uma maior ativação do giro angular esquerdo, estrutura associada ao autoconceito associado ao jogo, o que sugere que a identificação com o seu avatar pode ser um meio de compensação de ansiedade social. ${ }^{56}$

A diferente prevalência entre sexos tem sido explicada por fenómenos de vulnerabilidade genética e diferentes níveis de testosterona. Contudo, também foi sugerida a influência das estruturas cerebrais. ${ }^{57-59}$ Neste sentido, um estudo recente comparou indivíduos saudáveis e com PJI de ambos os sexos. Verificaram-se défices de atividade e conectividade nos indivíduos portadores desta perturbação, sendo mais significativos no sexo masculino do que no feminino. ${ }^{33}$

\section{COMORBILIDADES}

A PJI representa um desequilíbrio entre múltiplos circuitos cerebrais, como abordado na secção de Neurofi- siologia. Pela sobreposição com diversas doenças, a PJI pode ocorrer em simultâneo com patologias psiquiátricas e neurológicas.

Em 1981, foi descrito o primeiro caso de convulsões epiléticas induzidas por um videojogo. 60 Desde essa data, múltiplos estudos foram publicados a demonstrar a relação entre a epilepsia e os videojogos pela fotossensibilidade associada, com a elaboração de um consenso em 1994, no qual são explanados os fatores desencadeadores das convulsões epiléticas associadas aos videojogos e as recomendações para as evitar. ${ }^{61}$

Posteriormente, foi demonstrada uma relação entre os MMORPG e o desenvolvimento de crises epiléticas, as quais poderão ser provocadas por fatores adicionais para além da fotossensibilidade, tais como ansiedade, excitabilidade emocional, stress, fadiga e processamento cognitivo. 62

Estudos realizados indicam uma associação entre PJI, abuso de substâncias (nicotina, álcool e canábis) e problemas psicossociais. Para além disso, os indivíduos que sofrem de PJI apresentam maior prevalência de perturbação depressiva, ansiedade social, baixa autoestima, perturbação do sono, problemas de atenção, absentismo escolar e perturbação de oposição e desafio.7,63-65

A redução do tempo despendido na promoção da carreira académica é uma das principais consequências desta patologia que, não só limita a carreira profissional, como também cria conflitos entre adolescentes e pais. ${ }^{66}$

A potencial presença desta multiplicidade de comorbilidades na PJI deve alertar os profissionais de saúde para a necessidade do seu rastreio.

\section{TRATAMENTO}

Vários planos terapêuticos têm sido alvo de estudo na PJI. Contudo, pela maior prevalência nos adolescentes, os estudos incidem sobretudo nesta faixa etária, pelo que os autores abordarão apenas as intervenções avaliadas nos adolescentes. As intervenções terapêuticas podem ser divididas em três tipos principais: psicoterapia, farmacoterapia e outras terapias.

\section{PSICOTERAPIA}

A psicoterapia é um formato de tratamento atual e aprovado em diversas patologias psiquiátricas, tais como perturbações de humor, obsessivo-compulsiva, entre outras. ${ }^{67}$ A Terapia Cognitivo Comportamental (TCC) consiste numa intervenção psicossocial focada na alteração de distorções cognitivas e comportamentos, com vista à melhoria da regulação emocional e desen- 
volvimento de estratégias para resolução de problemas atuais. ${ }^{68}$ A sua aplicação demonstrou uma redução significativa na gravidade dos sintomas após um período de 6 semanas de tratamento. ${ }^{69}$ Num grupo diagnosticado concomitantemente com perturbação depressiva, verificou-se uma redução dos sintomas de PJI, avaliados pelo Young Internet Addiction Scale (YIAS) e pelo tempo médio de jogo, com a administração conjunta de TCC e bupropiom comparativamente ao bupropiom isolado. ${ }^{70}$ Todavia, a presença desta comorbilidade limita a determinação da eficácia real desta terapêutica.

O Programa Individualizado Psicoterapéutico para la Adicción a las Tecnologías de la Información y la Comunicación (PIPATIC) consiste num programa de intervenção individualizado para adolescentes com idades compreendidas entre os 12 e os 18 anos diagnosticados com PJI. Este programa tem como principais objetivos o uso adaptativo de videojogos e tecnologias de informação e o tratamento dos adolescentes de forma multidimensional. Apresenta uma duração de seis meses e integra diversas áreas de intervenção, sendo constituído por seis módulos, nomeadamente psicoeducacional, tratamento, intrapessoal, interpessoal, intervenção familiar e desenvolvimento de um novo estilo de vida. ${ }^{71}$ Comparativamente à TCC, ambos apresentaram uma redução dos sintomas de PJI, embora o grupo submetido a PIPATIC tenha apresentado também uma melhoria das capacidades intrapessoal e interpessoal e dos relacionamentos familiares. $^{72}$

A terapia familiar é uma terapia sistémica na qual a família constitui o grupo humano onde ocorrem as relações mais intensas e pressupõe a integração de vários elementos nas sessões. ${ }^{67}$ A aplicação deste plano terapêutico demonstrou uma redução da gravidade dos sintomas dos adolescentes. Todavia, a ausência de um grupo de controlo e a curta duração do tratamento limitam as potenciais conclusões. $^{73}$

O plano terapêutico eclético é composto por TCC, terapia familiar, terapia focada na solução e entrevista motivacional. A aplicação deste programa permitiu uma redução dos sintomas de PJI reportados pelos pais. Contudo, o mesmo não ocorreu com os adolescentes, fenómeno que pode estar associado à negação dos problemas e adesão relutante à terapêutica. ${ }^{74}$

Os campos residenciais terapêuticos envolvem programas de terapia ocupacional, terapia de exercício, TCC e atividades recreativas em que os adolescentes transitam para uma residência durante um período pré-determinado. Este plano terapêutico apresenta várias vantagens, nomeadamente manutenção da distância do ambiente de videojogo enquanto em terapia e experiência de relações interpessoais sem dispositivos eletrónicos. Um estudo verificou que, três meses após o término da terapia, o tempo global despendido em videojogos reduziu significativamente, embora os adolescentes mantivessem hábitos diários. ${ }^{75}$ Todavia, a maioria dos adolescentes apresentava concomitantemente perturbação do neurodesenvolvimento, principalmente Perturbação de Défice de Atenção e Hiperatividade (PHDA), o que limita as conclusões.

\section{FARMACOTERAPIA}

Uma das terapêuticas avaliadas foram os antidepressivos, nomeadamente bupropiom e escitalopram. Após 6 semanas de tratamento, ambos demonstraram efetividade no tratamento e controlo de sintomas de PJI. O bupropiom apresentou efetividade superior na melhoria da atenção e impulsividade dos adolescentes com PJ। em comparação com o escitalopram. Os adolescentes avaliados apresentavam como comorbilidade perturbação depressiva major, o que dificulta a avaliação da efetividade destes fármacos no tratamento da PJI isolada. ${ }^{76}$

O uso de metilfenidato e atomoxetina foi igualmente avaliado para esta patologia. Após 3 meses, ambos os fármacos tiveram como consequência a diminuição dos sintomas de PJI. Contudo, a diferença entre estes, nos instrumentos de avaliação utilizados, não era estatisticamente significativa. ${ }^{77}$ Para além disso, os adolescentes incluídos tinham sido diagnosticados previamente com PHDA, sendo uma das principais limitações do estudo.

\section{OUTRAS TERAPIAS}

A terapia equina é uma forma de tratamento utilizada em diversas áreas da medicina com vista à melhoria do estado emocional e capacidade de ligação social, tais como atraso global de desenvolvimento psicomotor e perturbação do espetro do autismo. ${ }^{78,79}$ Após 7 dias de terapia, os adolescentes com perturbação de ligação social diagnosticados com PJI apresentaram melhoria dos sintomas, o que poderá permitir a limitação da progressão de PJI e promover a sua resolução mais precoce. ${ }^{80}$

A participação num curso de leitura e escrita com abordagem de temáticas de MMORPG poderá melhorar os aspetos funcionais da expressão da linguagem e reduzir os sintomas de PJI através da modificação do processamento dos videojogos a nível cerebral. ${ }^{81}$

\section{DISCUSSÃO}

A sociedade está cada vez mais informatizada, consequência da maior difusão tecnológica. As crianças são cada vez mais expostas às tecnologias desde cedo, pelo 
que é importante a avaliação do impacto na sua saúde. $\bigcirc$ franco aumento da difusão da Internet promoveu uma nova modalidade de videojogos que apelam às massas. A perceção de que o seu uso indevido estaria a trazer consequências negativas na vida dos adolescentes fomentou interesse na investigação de uma potencial perturbação.

A definição de critérios diagnósticos pelo DSM-5 foi o fator que permitiu compreender a PJI como uma perturbação distinta. No entanto, esta proposta tem sido alvo de várias críticas. A própria classificação nosológica é incoerente, pelo que não exclui por completo jogos computorizados sem ligação à Internet. ${ }^{82}$ Além disso, os critérios foram emprestados da definição de perturbação de jogo e decididos com base em consensos, tal como o próprio limiar de 5 critérios. ${ }^{12}$ No entanto, apesar da sua validade diagnóstica ter vindo a ser questionada, vários estudos já a comprovaram¹7,83,84 e confirmaram a adequação do limiar diagnóstico. ${ }^{12,84}$ Também têm sido feitas críticas sobre os próprios critérios, tais como "fuga" e "preocupação", e a adequação do vocabulário à atualidade. Por outro lado, têm sido desenvolvidas propostas de melhoria, tais como a introdução de novos critérios e conceitos, tais como o "desejo". ${ }^{83,85,86}$

Recentemente, a CID-11 definiu os seus critérios e pressupostos para o diagnóstico da PJI e, por este motivo, apresenta algumas vantagens perante o DSM-5. A CID-11 é mais permissiva na duração da perturbação, dado que prevê que os casos mais graves possam ser diagnosticados antes de completar 1 ano. A sua definição é também mais restrita, uma vez que prevê uma clara distinção da perturbação do jogo online e offline. Porém, não está livre de limitações, tais como a condensação dos critérios de diagnóstico e a necessidade da presença concomitante dos três. Outra limitação, partilhada com o DSM-5, é a subjetividade dos critérios. A ausência de categorização do amplo espectro de alterações comportamentais e consequente repercussão nas atividades de vida diária perpetua o risco de diagnosticar incorretamente os grandes utilizadores com PJI.

Um dos grandes méritos do DSM-5 foi permitir elaborar os primeiros instrumentos de avaliação clínica específicos para PJI. Atualmente, a totalidade destes ainda são baseados nele. Todavia, a sua subjetividade supracitada veio promover a elaboração de várias e diferentes ferramentas diagnósticas com crescente utilização na investigação epidemiológica. Este facto condiciona uma heterogeneidade significativa que dificulta a avaliação da epidemiologia desta perturbação. Deste modo, é urgente o desenvolvimento de questionários validados com critérios de diagnóstico uniformes e limiares de rastreio padronizados, com vista à avaliação concisa do impacto da PJI a nível mundial.

A literatura revista parece ser bastante consensual em grupos de risco para desenvolvimento de PJI. Dada a prevalência crescente desde a infância até à idade adulta, é provável que esta doença progrida ao longo do desenvolvimento e maturação. Por outro lado, a prevalência de PJI no sexo masculino foi persistentemente superior, apesar de ainda não haver uma plausibilidade biológica assertiva. Assim, é urgente criar programas de rastreio dirigidos aos indivíduos mais jovens e do sexo masculino, tal como a outros grupos de risco que se venham a identificar. Neste sentido, os estudos em neurofisiologia contribuíram para a definição de grupos de risco através do reconhecimento de alterações em áreas implicadas na fisiopatologia da PJI.

Os estudos sobre a abordagem terapêutica apresentam limitações transversais à literatura revista, nomeadamente a prevalência de comorbilidades associadas, a curta duração dos tratamentos e a ausência de grupos de controlo. Dada a sobreposição fisiopatológica com outros distúrbios neuropsiquiátricos, as abordagens usadas na PJI são tendencialmente semelhantes. A TCC, o PIPATIC e a terapia familiar aparentam ser as opções mais promissoras, apesar de serem necessários estudos adicionais. A maior resistência a estes programas terapêuticos é a falta de cooperação dos adolescentes, característica da faixa etária. Por este motivo, será interessante o desenvolvimento de terapias mais direcionadas e adaptadas a estes indivíduos. No que diz respeito à farmacoterapia, até à data, foram estudadas duas classes: antidepressivos e fármacos direcionados para a PHDA. Apesar dos estudos reportarem eficácia dos respetivos agentes utilizados, não se pode descurar da presença de patologia concomitante que justificasse o seu uso. Consequentemente, são necessários estudos adicionais que avaliem a eficácia das várias terapêuticas, idealmente em cenários isolados.

A PJI é uma perturbação atual com impacto crescente. Por este motivo, o crescimento da investigação nesta área é significativo apenas nas últimas décadas. Por consequência, esta revisão destaca-se pela inclusão de diversas tipologias de trabalho com nível de evidência robusta, sem descurar os artigos originais que abordam um tema em permanente atualização. Outro ponto forte deste trabalho foi a limitação temporal, dado que os estudos prévios a 2013 raramente distinguiam a PJI como entidade distinta. Além disso, os estudos incluídos nesta revisão abordam todo o espectro etário afetado pela PJI, desde a criança até ao jovem adulto. No entanto, tal não se verifica na questão do tratamento, onde a literatura 
referente a adultos é escassa. Outro ponto fraco deste trabalho é a metodologia dos estudos epidemiológicos incluídos. Previamente supracitada, a sua heterogeneidade não nos permitiu averiguar o verdadeiro impacto mundial desta perturbação. Para isto, também contribui o seu predomínio ao nível do continente asiático, cujas questões linguísticas limitaram a disponibilidade de estudos publicados.

Em conclusão, a PJI é uma perturbação com tendência a aumentar, para a qual os clínicos devem estar alerta. É urgente atingir um consenso sobre os critérios de diagnóstico para permitir a implementação de instrumentos de rastreio padronizados e validados, com aplicação na prática clínica. Ademais, é igualmente necessária maior investigação de opções terapêuticas, de forma a orientar os indivíduos com esta perturbação da forma mais eficaz. Parte das medidas de saúde pública incluem a divulgação desta perturbação a pais e outros cuidadores de crianças e adolescentes e a responsabilização social das empresas de videojogos enquanto criadores do produto de consumo.

CONFLITOS DE INTERESSE: Os autores declaram não ter qualquer conflito de interesse na realização do presente trabalho.

FONTES DE FINANCIAMENTO: Não houve qualquer fonte de financiamento na realização do presente trabalho.

PROVENIÊNCIA E REVISÃO POR PARES: Não comissionado; revisão externa por pares.

CONFLICTS OF INTEREST: The authors declare that they have no conflicts of interest.

FINANCIAL SUPPORT: This work has not received any contribution, grant or scholarship.

PROVENANCE AND PEER REVIEW: Not commissioned; externally peer reviewed.

\section{REFERÊNCIAS}

1. Goldsmith JTT. Cathode-ray tube amusement device: United States Patent Office; 1948 [Accessed Feb 2019]. Available from: https://patents.google.com/patent/US2455992/en.

2. McPherson SS. Tim Berners-Lee: inventor of the world wide web. Minneapolis: Twenty-First Century Books; 2009.

3. Forsans E. The video game industry is adding 2-17 year-old gamers at a rate higher than that age group's population growth: Agence Française pour le Jeu Vidéo; 2011 [Accessed March 2019]. Available from: https://www.afjv.com/news/233_kids-and-gaming-2011.htm.

4. Gentile DA, Bailey K, Bavelier D, Brockmyer JF, Cash H, Coyne SM, et al. Internet gaming disorder in children and adolescents. Pediatrics. 2017;140:S81-S5. doi:10.1542/peds. 2016-1758H.

5. American Psychiatric Association. DSM-5 - manual de diagnóstico e estatística das perturbações mentais. Lisboa: Climepsi Editores; 2014.
6. Fam JY. Prevalence of internet gaming disorder in adolescents: A meta-analysis across three decades. Scand J Psychol. 2018;59:524-31. doi:10.1111/sjop.12459.

7. Desai RA, Krishnan-Sarin S, Cavallo D, Potenza MN. Video-gaming among high school students: health correlates, gender differences, and problematic gaming. Pediatrics. 2010;126:e1414-24. doi:10.1542/peds.2009-2706.

8. Newzoo. Global games market report. San Francisco: Newzoo; 2019.

9. King DL, Haagsma MC, Delfabbro PH, Gradisar M, Griffiths MD. Toward a consensus definition of pathological video-gaming: a systematic review of psychometric assessment tools. Clin Psychol Rev. 2013;33:331-42. doi:10.1016/j. cpr.2013.01.002.

10. Pontes HM, Griffiths MD. Assessment of internet gaming disorder in clinical research: past and present perspectives. Clin Res Regul Aff. 2014;31:35-48. doi:10.3109/10601333.2014. 962748.

11. Pontes HM, Kiraly O, Demetrovics Z, Griffiths MD. The conceptualisation and measurement of DSM-5 internet gaming disorder: the development of the IGD-20 test. PLoS One. 2014;9:e110137. doi:10.1371/journal.pone.0110137.

12. Lemmens JS, Valkenburg PM, Gentile DA. The internet gaming disorder scale. Psychol Assess. 2015;27:567-82. doi:10.1037/ pas0000062.

13. Vadlin S, Aslund C, Rehn M, Nilsson KW. Psychometric evaluation of the adolescent and parent versions of the gaming addiction identification test (GAIT). Scand J Psychol. 2015;56:72635. doi:10.1111/sjop.12250.

14. Muller KW, Janikian M, Dreier M, Wolfling K, Beutel ME, Tzavara $C$, et al. Regular gaming behavior and internet gaming disorder in european adolescents: results from a cross-national representative survey of prevalence, predictors, and psychopathological correlates. Eur Child Adolesc Psychiatry. 2015;24:565-74. doi:10.1007/s00787-014-0611-2.

15. Pearcy BT, Roberts LD, McEvoy PM. Psychometric testing of the personal internet gaming disorder evaluation-9: a new measure designed to assess internet gaming disorder. Cyberpsychol Behav Soc Netw. 2016;19:335-41. doi:10.1089/cyber.2015.0534

16. Jeromin F, RiefW, Barke A. Validation of the internet gaming disorder questionnaire in a sample of adult german-speaking internet gamers. Cyberpsychol Behav Soc Netw. 2016;19:4539. doi:10.1089/cyber.2016.0168.

17. Kiraly O, Sleczka P, Pontes HM, Urban R, Griffiths MD, Demetrovics Z. Validation of the ten-item internet gaming disorder test (IGDT-10) and evaluation of the nine DSM-5 internet gaming disorder criteria. Addict Behav. 2017;64:253-60. doi:10.1016/j.addbeh.2015.11.005.

18. Koo HJ, Han DH, Park SY, Kwon JH. The structured clinical interview for DSM-5 internet gaming disorder: development and validation for diagnosing IGD in adolescents. Psychiatry Investig. 2017;14:21-9. doi:10.4306/pi.2017.14.1.21.

19. Sigerson L, Li AY, Cheung MW, Luk JW, Cheng C. Psychometric properties of the chinese internet gaming disorder scale. Addict Behav. 2017;74:20-6. doi:10.1016/j.addbeh.2017.05.031.

20. Pontes HM, Griffiths MD. Portuguese validation of the internet gaming disorder scale-short-form. Cyberpsychol Behav Soc Netw. 2016;19:288-93. doi:10.1089/cyber.2015.0605.

21. World Health Organization. International classification of diseases 11th revision [homepage on the Internet] Geneva: World Health Organization; 2018 [Accessed March 2019]. Available from: https://icd.who.int/en. 
22. van Rooij AJ, Kardefelt-Winther D. Lost in the chaos: flawed literature should not generate new disorders. J Behav Addict. 2017;6:128-32. doi:10.1556/2006.6.2017.015.

23. Yuan K, Yu D, Cai C, Feng D, Li Y, Bi Y, et al. Frontostriatal circuits, resting state functional connectivity and cognitive control in internet gaming disorder. Addict Biol. 2017;22:813-22. doi:10.1111/adb.12348.

24. Kim SH, Baik SH, Park CS, Kim SJ, Choi SW, Kim SE. Reduced striatal dopamine D2 receptors in people with internet addiction. Neuroreport. 2011;22:407-11. doi:10.1097/WNR.0b013e328346e16e.

25. Hou H, Jia S, Hu S, Fan R, Sun W, Sun T, et al. Reduced striatal dopamine transporters in people with internet addiction disorder. J Biomed Biotechnol. 2012;2012. doi:10.1155/2012/854524.

26. Jorgenson AG, Hsiao RC, Yen CF. Internet addiction and other behavioral addictions. Child Adolesc Psychiatr Clin N Am. 2016;25:509-20. doi:10.1016/j.chc.2016.03.004.

27. Liu L, Yip SW, Zhang JT, Wang LJ, Shen ZJ, Liu B, et al. Activation of the ventral and dorsal striatum during cue reactivity in internet gaming disorder. Addict Biol. 2017;22:791-801. doi:10.1111/adb.12338.

28. Han DH, Kim SM, Bae S, Renshaw PF, Anderson JS. Brain connectivity and psychiatric comorbidity in adolescents with internet gaming disorder. Addict Biol. 2017;22:802-12. doi:10.1111/adb.12347.

29. Weinstein A, Lejoyeux M. New developments on the neurobiological and pharmaco-genetic mechanisms underlying internet and videogame addiction. Am J Addict. 2015;24:117-25. doi:10.1111/ajad.12110.

30. Dong G, Lin X, Potenza MN. Decreased functional connectivity in an executive control network is related to impaired executive function in internet gaming disorder. Prog Neuropsychopharmacol Biol Psychiatry. 2015;57:76-85. doi:10.1016/j. pnpbp.2014.10.012.

31. Weinstein A, Livny A, Weizman A. New developments in brain research of internet and gaming disorder. Neurosci Biobehav Rev. 2017;75:314-30. doi:10.1016/j.neubiorev.2017.01.040.

32. Luijten M, Meerkerk GJ, Franken $\mathrm{IH}$, van de Wetering BJ, Schoenmakers TM. An fMRI study of cognitive control in problem gamers. Psychiatry Res. 2015;231:262-8. doi:10.1016/j. pscychresns.2015.01.004

33. Sun $Y$, Wang $Y$, Han X, Jiang W, Ding W, Cao M, et al. Sex differences in resting-state cerebral activity alterations in internet gaming disorder. Brain Imaging Behav. 2019;13:1406-17. doi:10.1007/s11682-018-9955-4.

34. McTeague LM, Huemer J, Carreon DM, Jiang Y, Eickhoff SB, Etkin A. Identification of common neural circuit disruptions in cognitive control across psychiatric disorders. Am J Psychiatry. 2017;174:676-85. doi:10.1176/appi.ajp.2017.16040400.

35. Lin X, Dong G, Wang Q, Du X. Abnormal gray matter and white matter volume in 'internet gaming addicts'. Addict Behav. 2015;40:137-43. doi:10.1016/j.addbeh.2014.09.010.

36. Lee D, Namkoong K, Lee J, Jung YC. Abnormal gray matter volume and impulsivity in young adults with internet gaming disorder. Addict Biol. 2018;23:1160-7. doi:10.1111/adb.12552.

37. Zhou F, Montag C, Sariyska R, Lachmann B, Reuter M, Weber B, et al. Orbitofrontal gray matter deficits as marker of internet gaming disorder: converging evidence from a cross-sectional and prospective longitudinal design. Addict Biol. 2019;24:100-9. doi:10.1111/adb.12570.

38. Wang Z, Wu L, Yuan K, Hu Y, Zheng H, Du X, et al. Cortical thickness and volume abnormalities in Internet gaming disorder: evidence from comparison of recreational internet game users. Eur J Neurosci. 2018;48:1654-66. doi:10.1111/ ejn.13987.

39. Wang Y, Yin Y, Sun YW, Zhou Y, Chen X, Ding WN, et al. Decreased prefrontal lobe interhemispheric functional connectivity in adolescents with internet gaming disorder: a primary study using resting-state fMRI. PLoS One. 2015;10:e0118733. doi:10.1371/journal.pone.0118733.

40. Wang M, Zheng H, Du X, Dong G. Mapping internet gaming disorder using effective connectivity: a spectral dynamic causal modeling study. Addict Behav. 2019;90:62-70. doi:10.1016/j. addbeh.2018.10.019.

41. Kuss DJ, Griffiths MD. Internet gaming addiction: a systematic review of empirical research. Int J Ment Health Addict. 2012;10:278-96. doi:10.1007/s11469-011-9318-5.

42. Dong G, Potenza MN. A cognitive-behavioral model of Internet gaming disorder: theoretical underpinnings and clinical implications. J Psychiatr Res. 2014;58:7-11. doi:10.1016/j. jpsychires.2014.07.005.

43. Bjork JM, Smith AR, Chen G, Hommer DW. Mesolimbic recruitment by nondrug rewards in detoxified alcoholics: effort anticipation, reward anticipation, and reward delivery. Hum Brain Mapp. 2012;33:2174-88. doi:10.1002/hbm.21351.

44. Luijten M, Schellekens AF, Kuhn S, Machielse MW, Sescousse G. Disruption of reward processing in addiction: an image-based meta-analysis of functional magnetic resonance imaging studies. JAMA Psychiatry. 2017;74:387-98. doi:10.1001/jamapsychiatry.2016.3084.

45. Koepp MJ, Gunn RN, Lawrence AD, Cunningham VJ, Dagher A, Jones T, et al. Evidence for striatal dopamine release during a video game. Nature. 1998;393:266-8. doi:10.1038/30498.

46. Wang R, Li M, Zhao M, Yu D, Hu Y, Wiers CE, et al. Internet gaming disorder: deficits in functional and structural connectivity in the ventral tegmental area-accumbens pathway. Brain Imaging Behav. 2019;13:1172-81. doi:10.1007/s11682-0189929-6.

47. Kemph JP, Erikson EH. Identity, youth and crisis. New York: W. W. Norton Company, 1968. Behavioral Science. 1969;14:1549. doi:10.1002/bs.3830140209.

48. Ac-Nikolic E, Zaric D, Niciforovic-Surkovic O. Prevalence of internet addiction among school children in Novi Sad. Srp Arh Celok Lek. 2015;143:719-25. doi:10.2298/sarh1512719a.

49. Gonzalvez MT, Espada JP, Orgiles M, Morales A, Sussman S. Nicotine dependence as a mediator of project EX's effects to reduce tobacco use in scholars. Front Psychol. 2016;7:1207. doi:10.3389/fpsyg.2016.01207.

50. Higgins E. Self-discrepancy: a theory relating self and affect. Psychol Rev. 1987;94:319-40. doi:10.1037/ 0033-295X.94.3.319.

51. Strauman TJ. Self-discrepancies in clinical depression and social phobia: cognitive structures that underlie emotional disorders? J Abnorm Psychol. 1989;98:14-22. doi:10.1037/ 0021-843X.98.1.14.

52. Moretti MM, Higgins ET. Relating self-discrepancy to self-esteem: the contribution of discrepancy beyond actual-self ratings. J Exp Soc Psychol. 1990;26:108-23. doi:10.1016/ 0022-1031(90)90071-S.

53. Scott L, O'Hara MW. Self-discrepancies in clinically anxious and depressed university students. J Abnorm Psychol. 1993;102:282-7. doi:10.1037//0021-843x.102.2.282.

54. Ding WN, Sun JH, Sun YW, Chen X, Zhou Y, Zhuang ZG, et al. Trait impulsivity and impaired prefrontal impulse inhibition function in adolescents with internet gaming addiction revealed by a Go/No-Go fMRI study. Behav Brain Funct. 2014;10:20. doi:10.1186/1744-9081-10-20. 
55. Ko CH, Hsieh TJ, Wang PW, Lin WC, Yen CF, Chen CS, et al. Altered gray matter density and disrupted functional connectivity of the amygdala in adults with internet gaming disorder. Prog Neuropsychopharmacol Biol Psychiatry. 2015;57:18592. doi:10.1016/j.pnpbp.2014.11.003.

56. Dieter J, Hill H, Sell M, Reinhard I, Vollstadt-Klein S, Kiefer F, et al. Avatar's neurobiological traces in the self-concept of massively multiplayer online role-playing game (MMORPG) addicts. Behav Neurosci. 2015;129:8-17. doi:10.1037/bne0000025.

57. Li M, Chen J, Li N, Li X. A twin study of problematic internet use: its heritability and genetic association with effortful control. Twin Res Hum Genet. 2014;17:279-87. doi:10.1017/ thg.2014.32.

58. Mehta PH, Beer J. Neural mechanisms of the testosterone-aggression relation: the role of orbitofrontal cortex. J Cogn Neurosci. 2010;22:2357-68. doi:10.1162/jocn.2009.21389.

59. Goudriaan AE, Lapauw B, Ruige J, Feyen E, Kaufman JM, Brand $\mathrm{M}$, et al. The influence of high-normal testosterone levels on risk-taking in healthy males in a 1-week letrozole administration study. Psychoneuroendocrinology. 2010;35:1416-21. doi:10.1016/j.psyneuen.2010.04.005.

60. Rushton DN. "Space invader" epilepsy. The Lancet. 1981;317:501. doi:10.1016/S0140-6736(81)91888-2.

61. Binnie CD, Harding GFA, Richens A, Wilkins A. Video games and epileptic seizures - a consensus statement. Seizure. 1994;3:245-6. doi:10.1016/S1059-1311(05)80170-0.

62. Chuang YC. Massively multiplayer online role-playing game-induced seizures: a neglected health problem in internet addiction. Cyberpsychol Behav. 2006;9(:451-6. doi:10.1089/ cpb.2006.9.451.

63. van Rooij AJ, Kuss DJ, Griffiths MD, Shorter GW, Schoenmakers MT, Van de Mheen D. The (co-)occurrence of problematic video gaming, substance use, and psychosocial problems in adolescents. J Behav Addict. 2014;3:157-65. doi:10.1556/ JBA.3.2014.013.

64. Lo SK, Wang CC, Fang W. Physical interpersonal relationships and social anxiety among online game players. Cyberpsychol Behav. 2005;8:15-20. doi:10.1089/cpb.2005.8.15.

65. Swing EL, Gentile DA, Anderson CA, Walsh DA. Television and video game exposure and the development of attention problems. Pediatrics. 2010;126:214-21. doi:10.1542/peds.20091508.

66. Prensky M. Don't bother me mom-I'm learning! 1st ed. Minnesota: Paragon House; 2006.

67. Saraiva CB, Cerejeira J. Psiquiatria fundamental. Lisboa: Lidel; 2014.

68. Beck JS. Cognitive behavior therapy: basics and beyond. 2nd ed. New York: The Guilford Press; 2011.

69. Li H, Wang S. The role of cognitive distortion in online game addiction among chinese adolescents. Child Youth Serv Rev. 2013;35:1468-75. doi:10.1016/j.childyouth.2013.05.021.

70. Kim SM, Han DH, Lee YS, Renshaw PF. Combined cognitive behavioral therapy and bupropion for the treatment of problematic on-line game play in adolescents with major depressive disorder. Comput Human Behav. 2012;28:1954-9. doi:10.1016/j.chb.2012.05.015.

71. Torres-Rodriguez A, Griffiths MD, Carbonell X. The treatment of internet gaming disorder: a brief overview of the PIPATIC program. Int J Ment Health Addict. 2018;16:1000-15. doi:10.1007/s11469-017-9825-0.

72. Torres-Rodriguez A, Griffiths MD, Carbonell X, Oberst U. Treatment efficacy of a specialized psychotherapy program for internet gaming disorder. J Behav Addict. 2018;7:939-52. doi:10.1556/2006.7.2018.111.
73. Han DH, Kim SM, Lee YS, Renshaw PF. The effect of family therapy on the changes in the severity of on-line game play and brain activity in adolescents with on-line game addiction. Psychiatry Res. 2012;202:126-31. doi:10.1016/j.pscychresns.2012.02.011.

74. Pallesen S, Lorvik IM, Bu EH, Molde H. An exploratory study investigating the effects of a treatment manual for video game addiction. Psychol Rep. 2015;117(2):490-5. doi:10.2466/02. PR0.117c14z9.

75. Sakuma H, Mihara S, Nakayama H, Miura K, Kitayuguchi T, Maezono M, et al. Treatment with the self-discovery camp (SDiC) improves internet gaming disorder. Addict Behav. 2017;64:357-62. doi:10.1016/j.addbeh.2016.06.013.

76. Song J, Park JH, Han DH, Roh S, Son JH, Choi TY, et al. Comparative study of the effects of bupropion and escitalopram on Internet gaming disorder. Psychiatry Clin Neurosci. 2016;70:527-35. doi:10.1111/pcn.12429.

77. Park JH, Lee YS, Sohn JH, Han DH. Effectiveness of atomoxetine and methylphenidate for problematic online gaming in adolescents with attention deficit hyperactivity disorder. Hum Psychopharmacol. 2016;31:427-32. doi:10.1002/hup.2559.

78. del Rosario-Montejo O, Molina-Rueda F, Munoz-Lasa S, Alguacil-Diego IM. Effectiveness of equine therapy in children with psychomotor impairment. Neurologia. 2015;30:425-32. doi:10.1016/j.nrl.2013.12.023.

79. Llambias C, Magill-Evans J, Smith V, Warren S. Equine-assisted occupational therapy: increasing engagement for children with autism spectrum disorder. Am J Occup Ther. 2016;70:7006220040p1-p9. doi:10.5014/ ajot.2016.020701.

80. Kang KD, Jung TW, Park IH, Han DH. Effects of equine-assisted activities and therapies on the affective network of adolescents with internet gaming disorder. J Altern Complement Med. 2018;24:841-9. doi:10.1089/acm.2017.0416.

81. Kim PW, Kim SY, Shim M, Im C-H, Shon Y-M. The influence of an educational course on language expression and treatment of gaming addiction for massive multiplayer online role-playing game (MMORPG) players. Comput Educ. 2013;63:208-17. doi:10.1016/j.compedu.2012.12.008.

82. Paulus FW, Ohmann S, von Gontard A, Popow C. Internet gaming disorder in children and adolescents: a systematic review. Dev Med Child Neurol. 2018;60:645-59. doi:10.1111/ dmcn.13754.

83. Ko CH, Yen JY, Chen SH, Wang PW, Chen CS, Yen CF. Evaluation of the diagnostic criteria of internet gaming disorder in the DSM-5 among young adults in Taiwan. J Psychiatr Res. 2014;53:103-10. doi:10.1016/j.jpsychires.2014.02.008.

84. Muller KW, Beutel ME, Dreier M, Wolfling K. A clinical evaluation of the DSM-5 criteria for internet gaming disorder and a pilot study on their applicability to further internet-related disorders. J Behav Addict. 2019;8:16-24. doi:10.1556/2006.7.2018.140.

85. Kiraly O, Urban R, Griffiths MD, Agoston C, Nagygyorgy K, Kokonyei $\mathrm{G}$, et al. The mediating effect of gaming motivation between psychiatric symptoms and problematic online gaming: an online survey. J Med Internet Res. 2015;17:e88. doi:10.2196/ jmir.3515.

86. Rehbein F, Kliem S, Baier D, Mossle T, Petry NM. Prevalence of internet gaming disorder in german adolescents: diagnostic contribution of the nine DSM-5 criteria in a state-wide representative sample. Addiction. 2015;110:842-51. doi:10.1111/ add.12849. 